\title{
TI.50.1
}

\section{TIER Campus Success Program Call For Proposals (CFP)}

- PDF: TIER-Campus-Success-CFP-FINAL-20170725.pdf

- Text: TIER-Campus-Success-CFP-FINAL-20170725txt.txt

\section{More Information}

\begin{tabular}{|c|c|}
\hline Repository ID & TI.50.1 \\
\hline Persistent URL & http://doi.org/10.26869/TI.50.1 \\
\hline Title & TIER Campus Success Program Call For Proposals (CFP) \\
\hline Authors & Ann West, Erin Murtha, Dean Woodbeck \\
\hline Sponsor & Ann West, AVP of Trust and Identity, Internet2 \\
\hline \multicolumn{2}{|l|}{ Review } \\
\hline Status & Preserve \\
\hline Publish Date & July 25,2017 \\
\hline DOI & $10.26869 /$ TI.50.1 \\
\hline \multicolumn{2}{|l|}{ Signature } \\
\hline Deprecated & No \\
\hline Future Review & None \\
\hline \multicolumn{2}{|l|}{ Supersedes } \\
\hline \multicolumn{2}{|l|}{ Format } \\
\hline \multicolumn{2}{|l|}{ Related Docs } \\
\hline \multicolumn{2}{|l|}{$\begin{array}{l}\text { Development } \\
\text { Location }\end{array}$} \\
\hline \multicolumn{2}{|l|}{ IP Framework } \\
\hline Subject Tags & TIER, Adoption \\
\hline Notes & \\
\hline
\end{tabular}

